\title{
COVID19 impact on nuclear medicine: an Australian perspective
}

\author{
Geoff Currie $^{1}$
}

Published online: 15 April 2020

(C) Springer-Verlag GmbH Germany, part of Springer Nature 2020

\section{Introduction}

The COVID19 pandemic will redefine the way we live. The immediate threat from COVID19 to health and to the nuclear medicine community is rapidly changing. It is useful to consider how COVID19 is shaping nuclear medicine practice globally. The COVID19 pandemic requires reconsideration of the risk:benefit equilibrium in nuclear medicine practice [1]. Here, a perspective from an Australian context is provided. Consideration should be given to the interplay between the resilience and crisis management skills developed during the multiple Australian 99Mo crises of 2018 and then 2019, and the challenge fatigue these events have produced. Casting an eye forward, the silver lining, if we can be brave enough to consider, might be more sustainable practices. The nuclear medicine profession is also gifted with inherent capabilities associated with radiation safety management that confer agility in the changing landscape; radiation control provides an infection control barrier in practice, ALARA (as low as reasonably achievable) principles are transferrable, foundations of radiation safety (time, distance and shielding) translate to infection control and the concept of justification, optimisation and limitation can provide immediate guidance for decision making in the COVID19 crisis.

\section{Impact on practice}

Across Australia, at the time of writing, society was in lockdown. Only essential travel was permitted. Staff, who could work at home, were required to do so. Public gatherings of more than 2 people were outlawed and fines issued for

This article is part of the Topical Collection on Infection and Inflammation

Geoff Currie

gcurrie@csu.edu.au

1 Charles Sturt University, Wagga Wagga, NSW, Australia breaches. Crossing state borders was banned. The downstream effects of this have seen a reduction in demand for nuclear medicine services; however, this was already being experienced with the implementation of urgent/acute only services in many departments. During the last week of March, centralised pharmacies had experienced a 30\% reduction, $50 \%$ in larger centres like Sydney and only $25 \%$ in rural areas. Referrals had significantly reduced for sites remaining on full service with a representative example being a major site where general practitioner referrals reduced by $60 \%$ and specialist referrals by $30 \%$ for the last week of March. One report indicated a $90 \%$ reduction in general practitioner referrals to a normally busy private hospital.

This scenario raises a number of questions. What tools are used to determine which patients are sufficiently urgent to warrant scanning during the crisis? The process varies substantially across the sector. Furthermore, scans are not simply postponed as there is no clear point where lockdown and recommencement of usual services will commence. Does this change the threshold we apply to triaging an urgent scan? Postponement really represents cancellation. Does an urgent or acute scan in a patient positive for COVID19 or presenting with symptoms change triage? Do symptomatic patients get rescheduled until after confirmatory testing or are they redirected to other services?

Rosenthal [2] reminds us that the four pillars of bioethics are autonomy, beneficence, non-maleficence and justice. In this time of crisis, decisions should be grounded in beneficence, non-maleficence and equity; the patient themselves are perhaps not in the best position to determine urgency but in many cases, autonomous decisions not to attend appointments have been made. Rosenthal [2] suggest that deprioritisation should be on the basis of:

- Availability of reasonably or equally beneficial alternatives;

- Patients with limited life expectancy who are unlikely to benefit;

- Patients not likely to be compliant with interventions; nullifying the value of the nuclear medicine procedure. 
These are decisions Australian nuclear medicine teams are grappling with on a daily basis.

The mean age of Australian nuclear medicine patients is over 60 years of age and patients generally present with multiple comorbidities. The typical nuclear medicine patient is not only high risk for contracting COVID19 but is the most vulnerable to dire or fatal outcomes. This, in itself, justifies limiting elective and non-urgent procedures. It also demands specific strategies to limit the risk of transmission to all patients and staff, but in particular our most vulnerable. Strategies adopted in variable combinations in some Australian nuclear medicine departments include, without being limited to:

- Single patient waiting rooms with patients arriving no earlier than 5 min prior to their appointment.

- Adjustments to workflow so that larger gaps between patients decrease the risk of overlap.

- Reinforcement of social distancing through scheduling and seating throughout departments.

- Patients instructed to call when arriving and wait in their vehicle until a return call advises their appointment is open.

- Removal of refreshments, magazines, child toys and similar from the waiting room.

- Reduction in paperwork by use of electronic referrals and single use pens.

- Where possible, leaving doors open to avoid the need for using door handles.

- Hand sanitiser for all on entry to the department with appropriate signage providing sanitising instructions and other measures in place.

- Minimise cross-contamination of equipment by having single users for specific computers or terminals and radiopharmacy.

- Regular and thorough cleaning and sanitisation of all surfaces including handles, cameras and other equipment.

- Frequent hand sanitisation for staff including between patients, and between patient interaction and operation of equipment or movement to other areas.

- Use of personal protective equipment (PPE) where available, including gloves, gowns and face masks.

- Multi-camera departments have reserved specific equipment for positive or symptomatic patients and other equipment for negative and asymptomatic patients to avoid cross-contamination. Staff rostering to specific equipment is also required to minimise cross-contamination.

- All equipment is decontaminated between patients and wider booking times allow air recirculation and refresh between patients.

- Excluding, unless essential for patient support or communication, family or friends accompanying the patient for their appointment and scan.

- Sites managing symptomatic patients have inpatients delivered direct to the scanning room (no waiting room) as would be the case for the above-mentioned outpatients waiting in their vehicle.

- Afternoon bookings for COVID19 positive or symptomatic patients reduce the risk to negative patients.

- Use of disposable tourniquets for radiopharmaceutical injection.

- The use of auto-injectors substantially reduces the time staff are with COVID19-positive patients.

- All stress testing being performed with pharmacologic stress.

- Diverting all lung scans to CT pulmonary angiography (CTPA).

Over the last decade, there has been a redefining of the work function of the nuclear medicine technologist with diagnostic CT not only being part of the scope of practice but the foundation qualifications. SPECT/CT and PET/CT systems are frequently used as overflow for diagnostic CT when caseload on the stand-alone CT scans is excessive. One of the early responses to COVID19 imaging with chest CT in some Australian departments was to shunt COVID cases to nuclear medicine. This reflected the larger role CT plays in urgent and acute patients and the decreasing caseloads in nuclear medicine during the crisis. This approach allowed the high-volume diagnostic CT scanner to be a COVID19-free zone for negative patents with the lower volume symptomatic or COVIDpositive patients circulating through CT on hybrid nuclear medicine systems. While sensible, it has put additional risk, stress and strain on the nuclear medicine staff.

The other significant discussion point in Australia is the role of ventilation perfusion lung scanning. It is possible patients presenting for pulmonary embolism evaluation (an emergent study) are COVID19 positive. The lung scan appears less useful for evaluating COVID19 itself. Perfusion only scans allow a normal perfusion to clear the patient of pulmonary embolism and avoid the higher risk ventilation study. Concurrent COVID19 is likely to cause perfusion defects in the absence of pulmonary embolism [1]. There are concerns that the ventilation scan requires staff to spend more time close to the patient during a ventilation scan that is known to be a high risk of area contamination from patients breaking the apparatus seal or coughing. Furthermore, aerosol units are difficult to decontaminate. Departments performing SPECT/CT (common in Australia) may forgo the ventilation scan in favour of using the CT scan to represent airways and potential mismatch for pulmonary embolism diagnosis. Technegas is in widespread use in Australia and offers a number of clear advantages in the COVID19 patient. The ventilation procedure is faster, reducing time and improving compliance which in turn decreases the risk of room and staff contamination. The apparatus is single use without recirculation and so poses no risk between patients. In either case, subject to PPE availability, staff should wear a N95 mask, gown and 
gloves with all equipment being sanitised afterward. As previously outlined, in some departments, all lung scans have been routinely diverted to CTPA.

\section{Impact on workforce}

Nuclear medicine is a highly specialised discipline and staff are not readily substituted. It is essential, therefore, to protect the workforce to ensure the highly trained physicians, technologists and scientists are available to provide the life-saving and life-changing services required for acute or emergent situations. As discussed above, part of that solution is to limit services to those in most need.

To protect and preserve services and functions, a number of approaches have been used across Australia. Single centre departments, as described above, have split staffing between equipment with no cross-over to ensure a COVID19 patient team and a negative team. Some multicentre sites have split staff into teams to operate wholly at one site without cross-over. This allows protection of teams from COVID19 exposure in another team. For example, if a member of site team A was suspected of being COVID19 positive, the entire team would be quarantined but patients could continue to be serviced by site B team. In the larger public sector, a single hospital nuclear medicine department may be open to service patients while all others are in lockdown. If COVID19 is suspected amongst any of that team, that hospital service can be closed and the next public hospital team opened. Both of these scenarios are possible because of the downturn in patient demand for services.

The Australian nuclear medicine community relies on ANSTO for $99 \mathrm{Mo} / 99 \mathrm{mTc}$ supply. As seen in the 2018 and then 2019 mechanical failure-based supply crisis, the single supplier model has a degree of vulnerability. In any number of processes, if a single staff member was suspected of being COVID19 positive, the entire supply chain would be disrupted. ANSTO have split process staff into 2 teams with no cross-over between the teams. On the global scale, it is a small operation. While the reactor can produce $30 \%$ of global $99 \mathrm{Mo}$ demand, ANSTO only produces 130 generators weekly for domestic use. With the split teams comes inefficiency and this number has been reduced to 100 , forcing rationing measures in practice. Some demand has fallen for sites not operating and through lower use in centralised pharmacies. 99Mo production remains high which allows the fewer generators to be loaded with larger amounts of 99Mo that, for some departments, provides a 2-week supply, stretching the 100 weekly generators to meet national needs. If one of the teams is taken out of production due to suspected COVID19, clearly an acute shortage of generators will result (50 weekly).

A number of nuclear medicine staff are particularly vulnerable to COVID19 including older staff, those with health issues, those with diabetes and those Indigenous staff. It is a micro-version of the national dilemma; how do we restrict the entire population behaviour to protect the most vulnerable? Most centres simply redirect at risk staff into other areas like radiopharmacy or the COVID19-free imaging team. Working from home is not possible for clinically active staff but for some in management or reporting physicians, working remotely provides a good solution. Others have opted to take leave during the crisis, for some that is paid leave due to long service entitlements while others, it is unpaid. At the time of writing, I had not heard of any situations but no doubt some older staff have used COVID19 as the catalyst for retirement. Not all departments work in teams; with Australia being geographically sparse and vulnerable, staff often find themselves as the only operator for their region. A single example is the 244-km stretch of the well-populated region north of Sydney (Newcastle to Port Macquarie) that has a single nuclear medicine department (Taree), a single nuclear medicine technologist and remote physicians reporting. The technologist is 70 years old and no doubt torn between maintaining services to the community and protecting her own health and wellbeing.

The COVID19 crisis has seen significant disruption to professional life in Australia. With the geographic and professional isolation of nuclear medicine services and the population we serve, cancellation of conferences (e.g. ANZSNM2020), branch meetings and personal travel have increased the sense of professional isolation. RAINS (Rural Alliance in Nuclear Scintigraphy) has worked hard to keep rural and regional nuclear medicine communities and those professionally isolated in metropolitan areas connected and supported via FaceBook. RAINS has also used Zoom virtual conferencing to deliver professional development activities and to have social gatherings remotely (virtual version of the traditional Friday beer after work). The mental health and wellbeing of our communities will be challenged during this social and professionally isolating period.

Restrictions on non-essential travel have driven patients to reconsider their travel. In remote and rural communities, this might result in patients opting out of medically important nuclear medicine procedures. The small community of Wagga Wagga for example $(60,000$ population) services a very large health community of 280,000 people dispersed over 125,000 square kilometres. It is not uncommon for patients to drive several hours for services, often requiring time off work and overnight stays. COVID19 and associated restrictions have discouraged many remote patients to travel for services. In Western Australia, remote patients often fly to centralised nuclear medicine services and there are also fly in/fly out nuclear medicine services in several remote communities. With decreased domestic flight availabilities, these services, often to our most vulnerable Australians, have been limited.

There have also been significant challenges for nuclear medicine staff. The downturn in patient numbers has created acute financial pressure expected to be protracted beyond 6 months. The public sector (state Government owned) will accommodate this and given the decrease in service 
utilisation, while carrying salary costs, the remainder of the department budget is likely to be lower. The private sector is more vulnerable to fluctuations in demand and many staff face the prospect of redundancy, direct pay cuts, indirect pay cuts through fewer hours (4 days per week for all reduces salaries by $20 \%$ ), forced unpaid leave and forced paid leave (annual leave and long service leave). A number od sites have closed operations, many staff made redundantnad even more staff now working $40-60 \%$ of their usual hours. At the same time, access to and availability of PPE are becoming increasing limited. Some sites have reported running out of alcohol swabs to use for patient injections short term. Generally, a very difficult situation when staff feel both insecure in their employment and unsafe in their work environment.

\section{Impact on radiopharmaceutical supply}

ANSTO had 2 major (4 weeks each) maintenance periods scheduled in April and June of 2020. During this time, Australia would rely on imported $99 \mathrm{Mo}$ for the reactor maintenance period, and ANSTO 99Mo being shipped internationally and returned as generators for the generator production facility maintenance period. With borders closed, flights grounded and economic crisis globally, these periods would leave Australian nuclear medicine very vulnerable. ANSTO have delayed one of these periods and at the time of writing, working on solutions for the second. Furthermore, Australia relies on importation of a number of radionuclides (e.g. $123 \mathrm{I}, 67 \mathrm{Ga}, 201 \mathrm{Tl}$ and more) whose supply now confronts production and transport barriers. In Australia, we have already seen some limitations associated with cold kit importation. Domestically, significant disruption to the transport network (air and road) has caused widespread delays in deliveries and increased costs. For example, the Government has chartered a flight routinly to bring 123I mIBG into Australia from Japan.

\section{Other impacts}

Largely research and clinical trial recruitment has come to a stop due to COVID19. Multidisciplinary meetings have largely been moved to virtual platforms which in some cases enriches the meeting. Some physicians have moved to a remote reporting model which is not new but more widely used during the COVID19 crisis. The latter 2 of these impacts are different and perhaps challenging during the strain of the COVID19 crisis but may enhance outcomes and provide sustainability moving forward.

Student training has been a big challenge during the COVID19 crisis. At Charles Sturt University, we had a great deal of agility in student delivery which allowed very rapid conversion from face to face in class teaching to online delivery and student support. Students have been off campus since mid-March and staff are working from home. Not an easy feat given we have 19,000 students on campus across 6 major campuses and several smaller sites, and 2100 staff; but we already have over 20,000 students learning online. For nuclear medicine, the challenge was how to manage student clinical placements. At the time of writing, the position was that students already on clinical placement should stay on clinical placement if the clinical site continued to operate. This essentially related to residency students completing 36 weeks of training in their 4th year who had already been embedded in a clinical team for 3 months. The risk of bringing in new students to a team was considered unacceptable, especially for a short block of 4 weeks. Consequently, short placements for second and third year students had been postponed. This also recognises the decreasing demand for services, decreasing the learning opportunities for junior students. Indeed, most of these students requested delaying placement not for COVID19 reasons but to ensure the learning environment was richer and more diverse. The governing body (AHPRA) has been very responsive to the crisis and provided clear directions that if educational providers need to modify approved programs to traverse the COVID19 crisis, they do not need to provide approval if the learning outcomes for students do not change. The focus in managing student placements is on patient safety, staff safety, student safety, community safety and ensuring students demonstrate the requisite learning outcomes and capabilities.

\section{Summary}

COVID19 is the greatest challenge most of us will experience in our lifetimes. In nuclear medicine, the effects and implications are far reaching. Australia's geographic isolation provides a degree of both insulation from global crisis and vulnerability to ramifications. While both issues and solutions are shared across the globe during this time, there will also be some unique characteristics for individual international communities. The Australian perspective may provide a helpful perspective for our international family.

\section{Compliance with ethical standards}

This article does not contain any studies with human participants or animals.

Conflict of interest The author declares that there is no conflict of interest. 


\section{References}

1. Zuckier LS, Moadel RM, Haramati LB, Freeman LM. Diagnostic evaluation of pulmonary embolism during the COVID-19 pandemic. J Nucl Med. 2020. https://doi.org/10.2967/jnumed.120.245571.
2. Rosenthal MS. Ethical issues in radioisotope shortages: rationing and priority setting. J Nucl Med Technol. 2010;38:117-20.

Publisher's note Springer Nature remains neutral with regard to jurisdictional claims in published maps and institutional affiliations. 